\title{
Brief Report: Adults with Mild Autism Spectrum Disorders (ASD): Scores on the Autism Spectrum Quotient (AQ) and Comorbid Psychopathology
}

\author{
Cees Ketelaars $\cdot$ Ernst Horwitz $\cdot$ Sjoerd Sytema $\cdot$ \\ Johan Bos · Durk Wiersma · Ruud Minderaa • \\ Catharina A. Hartman
}

Published online: 26 March 2007

(C) Springer Science+Business Media, LLC 2007

\begin{abstract}
While knowledge about symptom presentation of adults with mild ASD, including comorbid psychopathology, is limited, referral of adults with suspected mild PDD is increasing. We report on pilot research investigating whether patients diagnosed with mild ASD $(n=15)$ and patients who were not diagnosed with ASD $(n=21)$ differed in terms of (a) AQ scores and (b) Axis I and II disorders, assessed by the SCAN and the IPDE. Additionally, AQ scores were compared with those from non-ASD patients referred to a general outpatient clinic $(n=369)$. The results showed very few differences between ASD patients and non-ASD patients. Self-report may not differentiate mild ASD patients from non-ASD patients and Axis I and II disorders seem equally prevalent among these two groups.
\end{abstract}

Keywords Autism Spectrum Quotient · Adult Autism Spectrum Disorders · PDDNOS · Comorbidity · Personality disorders

\section{Introduction}

Although there is consensus (APA, 1994) about DSM IV criteria for children and adolescents with Autistic Disorder and Asperger's disorder, the largest category, PDDNOS, remains difficult to classify. The latter is even more true for adult patients since the DSM-IV criteria are not formulated

C. Ketelaars

Autism Team North Netherlands, Groningen, The Netherlands

C. Ketelaars $(\square) \cdot$ E. Horwitz $\cdot$ S. Sytema

J. Bos - D. Wiersma - R. Minderaa - C. A. Hartman

University Medical Center Groningen (UMCG), Hanzeplein 1,

P.O. Box 660, Groningen 9700 AR, The Netherlands

e-mail: c.ketelaars@accare.nl with adult age in mind (Gillberg, 1998; Tamtam, 1991; Vermeulen, 2002). The Autism Diagnostic Interview Revised (ADI-R, Lord, Rutter, \& Le Couteur, 1994) and the Autism Diagnostic Observation Schedule-Generic (ADOS-G; Lord et al., 2000) are validated only for children and adolescents. Follow-up studies of adolescents and adults with autism indicate that a slow decrease in symptoms across time occurs in a small group, particularly the less severely affected patients (Seltzer, Shattuck, Abedutto, \& Greenberg, 2004). However, there is a lack of knowledge about adults with mild ASD, i.e., Asperger's Disorder and PDDNOS.

In light of this limited knowledge on symptom presentation in adults, the present paper reports on a pilot research-project that was done with two aims: (a) to test the Autism Spectrum Quotient (AQ; Baron-Cohen, Wheelwright, Skinner, Martin, \& Clubley, 2001) as an instrument for screening adult non-mentally retarded patients for ASD. We hypothesised that scores would be higher for an ASD group than for two non-ASD comparison groups; (b) there is "a clinical feeling" that mild ASD symptoms often mix with other disorders (e.g., Obsessive Compulsive Disorder or Schizoid Personality Disorder). Therefore, in patients referred to an ASD expertise-centre, prevalences of Axis I and II comorbid disorders were estimated to test the hypothesis that certain diagnoses are more prevalent among ASD patients than non-ASD patients.

\section{Method \\ Participants}

Two groups of patients were investigated; the first group $(n=36)$ consisted of patients referred to the "Autism 
Team North Netherlands" (ATN). The ATN is the ASD outpatient center of expertise for the three Northern provinces of the Netherlands ( 1.5 million inhabitants). On a yearly basis, about 80 new adult patients are referred for diagnostic evaluation and (advice for) treatment. Since the ATN is a "third line" center of expertise, all patients were referred by psychiatrists and psychologists, working in the field of adult (but not forensic) psychiatry. Only patients with parents who were able to give the required information were included in the present study. They were 18 years or older, with an estimated IQ higher than 80 . Over a span of 10 months from February 2003 through October 2003, 60 eligible patients were asked to participate, of which 35 patients and their parents consented. The patients were randomly assigned to two experienced (child) psychiatrists (CK or EH) for diagnostic assessment.

The second group $(n=369)$ consisted of patients from the same region, who were referred in this same period to the general outpatient psychiatric clinic of the University Medical Centre Groningen. This group from the general outpatient clinic (GOC) enabled us to compare the scores on the AQ screening instrument.

\section{Instruments}

The clinical standardized diagnostic protocol that was used in the intake of ATN-referred patients consisted of a semistructured interview (taken from at least one of the parents), and observations from the ADI-R, ADOS-G, as well as clinical experience. Actual ASD symptoms were translated in the interview as much as possible to the adultworld situation. Questions about the past were used to reconstruct the developmental history of the patient as adequately as possible. The information derived from the diagnostic protocol was subsequently used to score the DSM-IV criteria on ASD. Intelligence was assessed by using a short version of the "Groninger Intelligentie Test" (GIT, Luteijn, 1966).

For classification of PDDNOS, the minimal amount of positive DSM-IV criteria was two, of which at least one derived from the social interaction domain. For Asperger's Disorder at least two items on the social domain and at least one item on the stereotypic domain needed to be present with no early delay of language. For High Functioning Autism at least six items were needed to be present, with at least two items on the social domain and at least one positive item on each of the other two domains, with onset of abnormal functioning before the age of three years.

The reliability of the diagnostic protocol was tested by assessing a series of seven patients simultaneously by two experienced (child) psychiatrists (CK and $\mathrm{EH})$. These independent classifications differed only on one patient
(PDDNOS versus non-ASD). The seven patients were not included in the present study, which started after having determined diagnostic reliability.

The AQ was translated in Dutch and was named AQ-D (Dutch). This self-report screening questionnaire has 50 items, which score on 5 domains of behavior: social skill, attention switching, attention to detail, communication, and imagination. Each domain has a maximum score of 10 (for details on this instrument, see Baron-Cohen et al., 2001) The AQ-D was completed by the patient before the clinical procedure started. The investigators were blind to the outcome of AQ-D scores. Internal consistency reliability estimates (Cronbach's $\alpha$ ) for AQ subscales were acceptable for the ASD-group but somewhat on the lower side for the non-ASD groups. Averaged across the five subscales Cronbach's $\alpha$ was .67 for the ASD group, .62 for the nonASD group referred to the ATN, and .59 for the GOC group. For the total scale internal consistency reliability was acceptable in all three groups: i.e., .85, .82, and .79, respectively.

Present and life-time comorbidity were assessed by using the Schedules for Clinical Assessment in Neuropsychiatry (SCAN-2.1, Giel \& Nienhuis, 1996; World Health Organisation, 1992) and the International Personality Disorder Examination (IPDE, Loranger et al., 1994). IQ, SCAN and IPDE assessments were done by a psychologist (JB) who was blind to the outcome of the ASD psychiatric diagnosis. JB was formally trained by the Groningen WHO Training Center for administering the SCAN.

\section{Statistical Analysis}

Chi-square tests and analyses of variance were conducted to analyze possible differences between the groups.

\section{Results}

Fifteen of the 36 patients were diagnosed with ASD. In this ASD-group, 10 patients classified as PDDNOS, 4 patients as Asperger's Disorder and 1 patient as High Functioning Autism. The number of positive DSM-IV items ranged from 2 to 8 , with an average score of 3.8.

Table 1 shows gender, age, and IQ of ASD and nonASD patients. Also included in this table are gender and age of the GOC patients. The significant differences between the groups were (a) the younger age of the ASD patients relative to the other patients groups; (b) the younger age of the non-ASD group referred to the ATN relative to the GOC patients; and (c) the predominance of males in the ATN-referred patients compared with the GOC patients. 
Table 1 Characteristics of participants

\begin{tabular}{llll}
\hline Diagnosis & ASD $(n=15)$ & No ASD $(n=21)$ & $\begin{array}{l}\text { General outpatient clinic } \\
(n=369)\end{array}$ \\
\hline Male/female $^{\mathrm{a}}$ & $m=12, f=3$ & $m=18, f=3$ & $m=180, f=189$ \\
Average age $^{\mathrm{b}}$ (range, sd) & 22 years $(18-24,5)$ & 27 years $(18-55,9)$ & 35 years $(14-73,11)$ \\
Mean IQ (sd) & $104(10)$ & $105(12)$ & \\
\hline
\end{tabular}

a Difference between GOC group and non-ASD group (chi-square $=14.2, \mathrm{df}=1, p<0.0001$ ); difference between GOC group and ASD group (chi-square $=5.8, \mathrm{df}=1, p<0.05)$; no difference between ASD group and non-ASD group

b GOC group differs from non-ASD group $(p<0.01)$ and from ASD group $(p<0.0001)$; no difference between ASD group and non-ASD group

Table 2 shows AQ-D sub-domain and total scores for the three groups. Generally, there were no significant differences between the three groups. The one significant difference was on the sub-domain Communication between the ASD group and the general outpatient group. Further, within the ASD group, average AQ-D scores varied with the severity of ASD: High Functioning Autism: $31(n=1)$; Asperger's Disorder: $24 \quad(n=4)$, and PDDNOS: 21 $(n=10)$. In light of the small sample sizes the latter differences were not tested for statistical significance.

Table 3 reports Axis I diagnoses in ASD and non-ASD patients as assessed by the SCAN. With one exception, there were no differences in either Axis I or Axis II diagnoses between the two groups. This held for both actual and lifetime (not reported) Axis I diagnoses. The one exception was that more patients were diagnosed with actual psychotic disorder NOS in the non-ASD group than in the ASD group. When we aggregated separate categories into more broad diagnostic categories, i.e., anxiety disorders, psychotic disorders, and mood disorders, there were no differences between the two groups in past-month or lifetime diagnoses either, except for actual psychotic disorders (results not shown).

Table 4 reports Axis II diagnoses as assessed by the IPDE. ASD and non-ASD patients did not differ on indi-

Table 2 Mean scores AQ-D (sd) and ANOVA tests for differences in mean scores

\begin{tabular}{llcl}
\hline & $\begin{array}{l}\text { ASD } \\
(n=15)\end{array}$ & $\begin{array}{l}\text { No ASD } \\
(n=21)\end{array}$ & $\begin{array}{l}\text { General outpatient } \\
\text { clinic }(n=369)\end{array}$ \\
\hline $\begin{array}{l}\text { Social skill } \\
\text { Attention } \\
\quad 5 \text { switching }\end{array}$ & $5.0(2.8)$ & $4.2(2.6)$ & $4.1(2.6)$ \\
$\begin{array}{l}\text { Attention to } \\
\quad \text { detail }\end{array}$ & $3.5(2.3)$ & $4.4(2.2)$ & $4.2(2.3)$ \\
$\begin{array}{l}\text { Communication* } \\
\text { Imagination }\end{array}$ & $4.5(2.1)$ & $3.5(2.2)$ & $2.8(1.8)$ \\
Total & $4.4(2.4)$ & $4.3(2.0)$ & $3.5(1.9)$ \\
& $22.5(8.4)$ & $21.8(7.6)$ & $19.9(7.0)$
\end{tabular}

* Significant difference between ASD group and GOC group at $p<0.01$ vidual Axis 2 diagnoses. Also, when we tested whether ASD and non-ASD groups differed with regard to the total number of patients with any complete, partial, or either of these two, Axis 2 disorders (bottom two rows, Table 4), we found no difference between the two groups.

\section{Discussion}

Thus far, publications about screening instruments for adult ASD use the Autism-Spectrum Quotient (AQ; Baron-Cohen et al., 2001) or the Autism Spectrum Disorder in Adults Screening Questionnaire (ASDASQ; Nylander \& Gillberg, 2001). With the help of the latter instrument, Nylander \& Gillberg (2001) and Chang et al. (2003) estimated a 1.4 and $0.6 \%$ ASD prevalence in a general psychiatry outpatient clinic for adults. The AQ was investigated by Baron-Cohen in 2001 in Asperger's

Table 3 SCAN-derived actual (past month) DSM IV Axis I diagnoses

\begin{tabular}{|c|c|c|}
\hline & $\begin{array}{l}\text { ASD } \\
(N=15) \\
n(\%)\end{array}$ & $\begin{array}{l}\text { No ASD } \\
(N=21) \\
n(\%)\end{array}$ \\
\hline No disorder & $7(47)$ & $7(33)$ \\
\hline $\begin{array}{l}\text { Mood disorder with psychotic } \\
\text { symptoms }\end{array}$ & $2(13)$ & - \\
\hline $\begin{array}{l}\text { Mood disorder without psychotic } \\
\text { symptoms }\end{array}$ & $2(13)$ & $3(14)$ \\
\hline Substance abuse & $3(20)$ & $2(10)$ \\
\hline Sleep disorders & $4(27)$ & $6(29)$ \\
\hline Psychotic disorder NOS & - & $4(19)^{*}$ \\
\hline Schizophrenia & - & - \\
\hline Social Phobia & $3(20)$ & $4(19)$ \\
\hline Panic attacks/Agoraphobia & $2(13)$ & $1(5)$ \\
\hline Other anxiety disorders & $1(7)$ & - \\
\hline Obsessive compulsive disorders & $1(7)$ & $1(5)$ \\
\hline Other disorders & $1(7)$ & $2(10)$ \\
\hline
\end{tabular}

* Significant difference between ASD group and no ASD group at $p<0.05$ 
Table 4 IPDE derived Axis II diagnoses by ASD status

\begin{tabular}{|c|c|c|c|c|}
\hline & \multicolumn{2}{|c|}{$\operatorname{ASD}(N=15)$} & \multicolumn{2}{|l|}{$\begin{array}{l}\text { No ASD } \\
(N=21)\end{array}$} \\
\hline & $\begin{array}{l}\text { Complete } \\
n(\%)\end{array}$ & $\begin{array}{l}\text { Partial } \\
n(\%)\end{array}$ & $\begin{array}{l}\text { Complete } \\
n(\%)\end{array}$ & $\begin{array}{l}\text { Partial } \\
n(\%)\end{array}$ \\
\hline Paranoïd & - & - & - & $1(5)$ \\
\hline Schizoïd & $1(7)$ & $2(13)$ & $1(5)$ & $1(5)$ \\
\hline Schizotypical & - & $1(7)$ & - & - \\
\hline Antisocial & - & $1(7)$ & - & - \\
\hline Borderline & $1(7)$ & - & - & $1(5)$ \\
\hline Avoidant & $1(7)$ & $1(7)$ & $2(10)$ & - \\
\hline Obsessive compulsive & - & - & $3(14)$ & $1(5)$ \\
\hline Personality disorder NOS & $1(7)$ & $2(13)$ & $3(14)$ & $1(5)$ \\
\hline Any personality disorder & $3(20)$ & $6(40)$ & $8(38)$ & $3(14)$ \\
\hline $\begin{array}{l}\text { Any personality disorder } \\
\text { partial or complete }\end{array}$ & \multicolumn{2}{|c|}{$7(47)$} & \multicolumn{2}{|c|}{$10(48)$} \\
\hline
\end{tabular}

Disorder or High Functioning Autism patients and compared with several subgroups in the general population, and was tested again in 2005 at the national diagnostic clinic for Asperger's Disorder (Woodbury-Smith, Robinson, Wheelwright, \& Baron-Cohen, 2005). The AQ differentiated the ASD patients adequately from healthy controls, and ASD patients from non-ASD patients.

The present study tried to replicate the findings of Baron-Cohen. However, our results showed that the AQ-D did not differentiate between an ASD group (predominantly PDDNOS) and two non-ASD patient groups. Only for the communication domain, the ASD patients had higher scores relative to the general outpatient group. It is of interest to mention here that this domain was also one of the two domains that differentiated parents of autistic patients from healthy controls (Bishop et al., 2004). The most probable explanation of this discrepant outcome is the fact that the Dutch patients were less severely affected than the British patients. The ASD patients but also the non-ASD patients in the Baron-Cohen papers had higher total mean scores on the AQ (for ASD: 35.8 and 35.6 and for nonASD: 26.2) than our patients (ASD: 22.5, non-ASD and general outpatients: 21.8 and 19.9). By comparison, British, non-psychiatric, control groups typically have total scores of around 16-17 (Baron-Cohen et al., 2001). Interestingly, the mean score on the recent Japanese version of the AQ was 29.4 in ASD patients and 22.2 in non-psychiatric controls (Kurita, Koyoma, \& Osada, 2005). The Japanese authors, like we, reasoned that part of the Japanese patients may have been less severely affected relative to the British patients. They also argued that it is possible that autistic related behavior as assessed by the AQ is more prevalent in the Japanese population than in the British. In the absence of the actual diagnoses of the non-ASD patients in both the British and one of the Dutch samples, it remains difficult to pinpoint why scores between the British and Dutch non-ASD patients (26.2 and 21.8/19.9, respectively) differ. The absence of differences between scores of the AQ-D in the present study suggests that there might be "ASD-like" symptoms, as felt by the patients themselves, present in the non-ASD patients in the ATNreferred group and in the patients of the general outpatient clinic, and revealed by self-report. Indeed, the individuals in the non-ASD group were referred to the ATN for the possible presence of an ASD diagnosis, so ASD-like symptoms must have been voiced by these patients even if these could not be diagnosed as such on the basis of the standardized diagnostic protocol. In sum, although there are still many open questions, for example about differences in scores cross-culturally, the present data suggest that self-report questionnaires are not adequate for differentiating less severe ASD patients from other patient groups. Another study is needed to investigate the merits of an alternative approach to this problem; i.e., a parents/ caregivers questionnaire as a screening tool for adult ASD.

Our second aim was to look into possible differences in comorbidity of Axis I and Axis II DSM IV classifications in ASD and non-ASD groups. This because of the notion that mild ASD symptoms often mix with symptoms of other disorders such as Obsessive Compulsive Disorder or Schizoid Personality Disorder. There is a scarcity of research on this topic. For example, (comorbid) diagnoses of the British patients were not reported in the paper of Woodbury-Smith et al. (2005). For the Dutch, ATN-referred group, however, we took a systematic approach to diagnosing DSM IV Axis I and II disorders. The results indicated that, except for Psychotic Disorder NOS which was diagnosed in roughly $20 \%$ of the non-ASD group and not in the ASD group, there were no significant differences in the pattern of diagnoses between the ASD and non-ASD patients. Possibly, the difference in Psychotic Disorder partly accounts for the referral to the ATN and for the "ASD-like" symptoms in these patients as discussed in the above paragraph. However, the general picture indicated by our data is that clinical accounts of a relatively high prevalence and/or a specific profile of comorbid disorders in the ASD group relative to the non-ASD group could not be confirmed.

There are important limitations to this study, among which is the small number of patients in the ATN-referred group. This major drawback precludes any definite conclusions. Of note is further that the ASD patients in this investigation belonged to the less-severe side of the spectrum. This makes it difficult to compare results with the investigations by Baron-Cohen and collegues. Clinical experience indicates that ASD patients, compared to 
non-ASD patients, benefit more from treatment by structuring, long-term repetitive treatment, and adaptations of the environment. This may hold even more when comorbid conditions are present. Since the prevalence of patients with less severe ASD is relatively high compared to the more severe ASD categories, efficient and valid screening of this group and charting possible comorbidity is consequently an even more important issue. The present study provided a modest contribution in improving our knowledge in this direction; clearly, much more research is needed.

Acknowledgments We are grateful to the Stichting tot Steun VCVGZ in Arnhem for supporting this project. We thank Dr. BaronCohen for allowing us to translate the AQ into Dutch.

\section{References}

American Psychiatric Association. (1994). Diagnostic and statistical manual of mental disorders (4th ed., DSM-IV). Washington, DC: American Psychiatric Press.

Baron-Cohen, S., Wheelwright, S., Skinner, R., Martin, J., \& Clubley, E. (2001). Autism-spectrum quotient (AQ): Evidence from Asperger syndrome/high-functioning autism, males and females, scientists and mathematicians. Journal of Autism and Developmental Disorders, 31, 5-17.

Bishop, D. V. M., Mayberry, M., Maley, A., Wong, A., Hill, W., \& Hallmayer, J. (2004). Using self-report to identify the broad phenotype in parents of children with autistic spectrum disorders: A study using the Autism-Spectrum Quotient. Journal of Child Psychology and Psychiatry, 45, 1431-1436.

Chang, H., Juang, Y., Wang, W., Huang, C., Chen, C., \& Hwang, Y. (2003). Screening for autism spectrum disorder in adult psychiatric outpatients in a clinic in Taiwan. General Hospital Psychiaty, 25, 284-288.

Giel, R., \& Nienhuis, F. J. (1996). SCAN-2.1: Schedules for clinical assessment in neuropsychiatry (vragenschema's voor klinische beoordeling in neuropsychiatrie). Geneva/Groningen: WHO.

Gillberg, C. (1998). Asperger and high-functioning autism. British Journal of Psychiatry, 172, 200-209.
Kurita, H., Koyoma, T., \& Osada, H. (2005). Autism spectrum quotient-japanese version and its short forms for screening normally intelligent persons with pervasive developmental disorders. Psychiatry and Clinical Neuroscience, 59, 490-496.

Loranger, A. W., Sartorius, N., Andreoli, A., Berger, P., Buchheim, P., Channabasavanna, S. M., Coid, B., Dahl, A., Diekstra, R. F. W., Ferguson, B., Jacobsberg, L. B., Mombour, W., Pull, C., Ono, Y., \& Regier, D. A. (1994). The International Personality Disorder Examination: IPDE. The WHO/ADAMHA International pilot study of personality disorders. Archives of General Psychiatry, 51, 215-224.

Lord, C., Rutter, M., \& Le Couteur, A. (1994). Autism Diagnostic Interview-Revised: A revised version of a diagnostic interview for caregivers of individuals with possible pervasive developmental disorders. Journal of Autism and Developmental Disorders, 24, 659-685.

Lord, C., Risi, S., Lambrecht, L., Cook, E. H. Jr., Leventhal, B. L., DiLavore, P. C., Pickles, A., \& Rutter, M. (2000). The autism diagnostic observation schedule-generic: A standard measure of social and communicative deficits associated with the spectrum of autism. Journal of Autism and Developmental Disorders, 30, 205-223.

Luteijn, F. (1966). A new abbreviated Groninger Intelligence Test. Nederlands Tijdschrift Psychologie, 21, 675-82.

Nylander, L., \& Gillberg, C. (2001). Screening for autism spectrum disorders in adult psychiatric outpatients: A preliminary report. Acta Psychiatrica Scandinavica, 103, 428-434.

Tantam, D. (1991). Asperger syndrome in adulthood. In U. Frith (Ed.), Autism and Asperger syndrome (pp. 147-183). Cambridge/New York: Cambridge University Press.

Vermeulen, P. (2002). Beter vroeg dan laat en beter laat dan nooit: De onderkenning van autisme bij normaal tot hoog begaafde personen. (Better late than never: The diagnosis of autism in adults with a normal-IQ). Thesis: Rijksuniversiteit Groningen.

Seltzer, M. M., Shattuck, P., Abedutto, L., \& Greenberg, J. S. (2004). Trajectory of development in adolescents and adults with autism. Mental Retardation and Developmental Disabilities Research Reviews, 10, 234-247.

Woodbury-Smith, M. R., Robinson, J., Wheelwright, S., \& BaronCohen, S. (2005) Screening adults for asperger syndrome using the AQ: A preliminary study of its diagnostic validity in clinical practice. Journal of Autism and Developmental Disorders, 35, 331-335.

World Health Organisation (1992). Schedules for clinical assessment in neuropsychiatry. Geneva: WHO. 